\title{
Left Atrial Appendage Morphology in Patients with Non-Valvular Atrial Fibrillation
}

\author{
Sonya Joy, $M D^{1 *}$, Horst Sievert, $M D^{1,2}$, Stefan Bertog, $M D^{1,3}$, Timothy Betts, $M D^{4}$, \\ Neil Wilson, $\mathrm{MD}^{1,5}$, Saul Myerson, $\mathrm{MD}^{6}$ \\ ${ }^{1}$ CardioVascular Center, Frankfurt, Germany \\ ${ }^{2}$ Anglia Ruskin University, Cambridge and Chelmsford, UK \\ ${ }^{3}$ Interventional Cardiology Veterans Administration Medical Center, University of Minnesota, Minneapolis, USA \\ ${ }^{4}$ Oxford University Hospitals NHS Trust, Oxford, UK \\ ${ }^{5}$ Childrens Hospital Colorado, Colorado, USA \\ ${ }^{6}$ University of Oxford Centre for Clinical Magnetic Resonance Research, Oxford, UK
}

\begin{abstract}
Aims: Percutaneous left atrial appendage (LAA) occlusion has now become a suitable alternative to oral anticoagulation for stroke prevention in selected patients with atrial fibrillation (AF). However, LAA closure can be technically challenging and results suboptimal, in part due to variable left atrial anatomy.We aimed to characterize LAA morphology and identify potential anatomical pitfalls during LAA closure or LAA thrombus detection during transoesophageal echocardiography (TOE).

Methods and Results: 103 patients with AF underwent cardiac magnetic resonance angiography to assess pulmonary venous anatomy. Adequate imaging quality was present in 76 in whom LAA morphology was assessed. The majority of LAAs $(\mathbf{7 1 \% )}$ were anterolaterally directed and $82 \%$ were 'claw'-shaped. However, there was significant variation in anatomy and course in the remainder: $11 \%$ were anteverted, $9 \%$ laterally directed and $9 \%$ retroverted. The shape was cone-like in $8 \%$, fan-like in $5 \%$ and s-configured in $5 \%$ and there was significant variation in the curvature of the LAA body. While $66 \%$ had a single lobe, $30 \%$ were bilobed and $\mathbf{4 \%}$ trilobed; $\mathbf{9 0 \%}$ also had additional lobules.

Conclusion: Our results demonstrate the significant variability of LAA geometry in AF patients. This may have implications for future device design for percutaneous LAA occlusion. The variable anatomy may affect LAA
\end{abstract}

(C) 2017 Journal of Structural Heart Disease Published by Science International Corp. ISSN 2326-4004 thrombus detection with TOE emphasizing the importance of multiple views to ensure complete assessment. Copyright $\odot 2017$ Science International Corp.

\section{Key Words}

Left atrial appendage $•$ Left atrial appendage morphology - Left atrial appendage closure • Atrial fibrillation

\section{Introduction}

Stroke is the most devastating atrial fibrillation (AF)-related event. Non-valvular chronic AF is associated with a more than 5 -fold increase in stroke risk [1], with the left atrial appendage (LAA) as the site of thrombogenesis in more than $90 \%$ of stroke victims [2]. Although oral anticoagulation with warfarin reduces this risk by more than half $[3,4]$, only $50-70 \%$ of patients with $\mathrm{AF}$ who are eligible for anticoagulation actually receive it. Novel anticoagulants are at least as effective as warfarin, but a significant bleeding risk remains. Thus, alternative options for stroke prevention are needed, particularly for patients with contraindications to anticoagulation.

Percutaneous LAA closure has the advantage of obviating long-term anticoagulation. The PROTECT-AF trial demonstrated that percutaneous LAA

* Corresponding Author:

Horst Sievert, MD

Cardiovascular Center

Seckbacher Landstrasse 65, 60389 Frankfurt, Germany

Tel. +49 69 4603-1343, Fax: +49 69 4603-1343, E-Mail: horstsievertmd@aol.com 

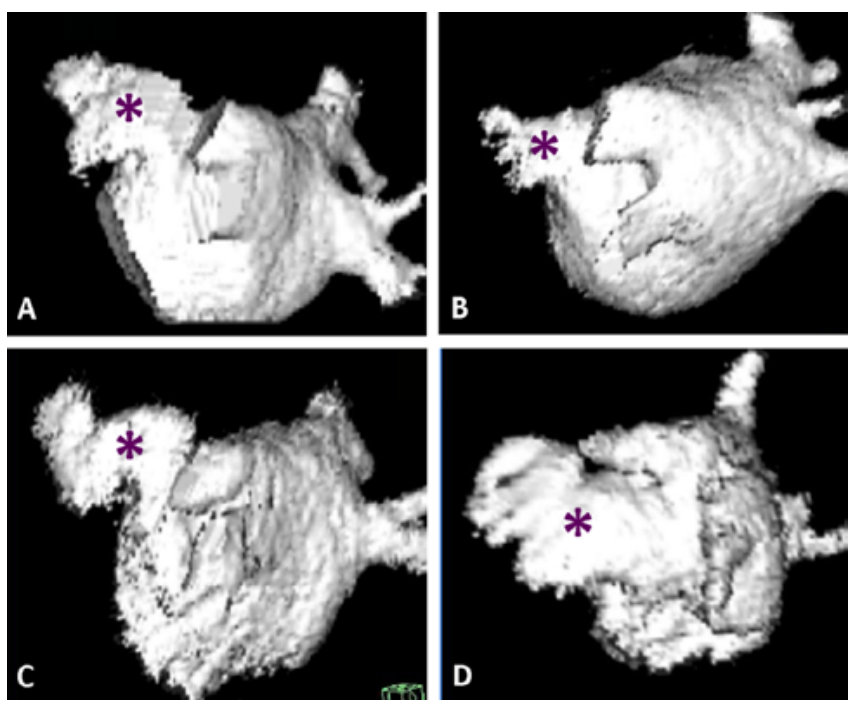

Figure 1. Representative left atrial appendage morphologies (three-dimensional surface rendered angiographic images). Panel A. Bi-lobed. Panel B. Fan-shaped with three small lobules. Panel C. s-configured. Panel D. Very large fan-shaped with secondary anterior lobe and multiple lobules. All images are viewed from a similar left postero-lateral position; the left atrial appendage is indicated by an asterisk (*).

closure is at least as safe and effective as anticoagulation with warfarin with regard to all-cause mortality and stroke risk [5]. LAA-occlusion devices have a fixed shape designed to provide an effective seal and stable positioning, but this may not take into account significant variability in LAA shape, orientation, and structure. Furthermore, regardless of the LAA closure device used, co-axial alignment of the delivery sheath within the appendage is crucial for safe and successful implantation, and this depends on the orientation of the LAA. Suboptimal alignment of the delivery system within the appendage may cause perforation $[5,6]$ and poor final device position, potentially promoting residual leaks [7] and thrombus formation. These complications remain the Achilles heel of the procedure, partially offsetting its potential benefits.

In addition, variation in LAA anatomy may influence the detection of LAA thrombus during transoesophageal echocardiography (TOE). Thrombus located in accessory or retroverted lobes might escape recognition in the usual TOE views, which may partially explain the small incidence of stroke despite TOE interrogation of the LAA prior to cardioversion [8]. Hence, a good understanding of LAA anatomy and orientation is important. Several studies have highlighted variations in LAA anatomy, particularly focusing on LAA size, branches/lobes, or orifice diameter $[9,10]$. Few studies, however, have described LAA orientation and shape, which are important for device and delivery system design [11, 12].

Here, we examined the range of three-dimensional (3-D) anatomy and 2-D geometry of the LAA in patients with non-valvular AF to allow sheath configuration and device design improvements that facilitate delivery and deployment. In addition, recognition of unusual anatomical variants may prompt clinicians to interrogate the LAA in all dimensions, which could improve thrombus detection.

\section{Materials and Methods}

\section{Study Population}

The population consisted of 103 consecutive patients with non-valvular AF who underwent left atrial and pulmonary vein (PV) angiograms using cardiovascular magnetic resonance (CMR) for the purpose of PV isolation ablation (94\%), evaluation for possible PV stenosis after PV isolation ablation $(5 \%)$, or cardiac surgery (1\%). The 3-D datasets were processed to ensure good visualization of the LAA and PVs. Twenty-seven patients were excluded due to inadequate image quality. In the remaining 76 patients in whom the LAA was analyzed, $67 \%$ were male and $33 \%$ were female, with a mean age of $56 \pm 11$ years (range, $18-77$ years).

\section{CMR Scanning and Image Processing}

Scans were performed using a 1.5-Tesla magnetic resonance scanner (Siemens Avanto, Siemens Medical Imaging, Erlangen, Germany) at the University of Oxford Centre for Clinical Magnetic Resonance Research. After obtaining localizer images, 3-D contrast-enhanced MR angiograms were acquired using a spoiled gradient echo sequence in a coronal voxel positioned to include the whole left atrium and proximal PVs and timed to the first passage of gadolinium contrast in the left atrium following a test bolus of $2 \mathrm{~mL}$ gadolinium contrast. The sequence was acquired during a single 20-30-s breath-hold and was not ECG-gated; scan parameters: TE, 1.1 $\mathrm{ms}$; TR, $3.0 \mathrm{~ms}$; flip angle, $25^{\circ}$; FoV, $360-400 \mathrm{~mm}$; slice thickness, $1.2 \mathrm{~mm}$; 96 slices per slab (slab thickness, $115 \mathrm{~mm}$ ); and iPAT factor, 3 (GRAPPA). Contrast enhancement was achieved with $0.15 \mathrm{mmol} / \mathrm{kg}$ body weight of gadodiamide (Omniscan:, GE Healthcare, Cleveland, OH, USA) administered via an ante-cubital vein at $6 \mathrm{~mL} / \mathrm{s}$ followed by a saline flush of $20 \mathrm{~mL}$ at the same injection rate. After acquisition, data were processed using Siemens Argus software to generate a 3-D surface-rendered image of the left atrium including the LAA. Surrounding structures, such as the aorta, right ventricle, and any residual pulmonary arteries were carefully edited out of the image. This 3-D model was used for assessment of LAA shape, orien- 


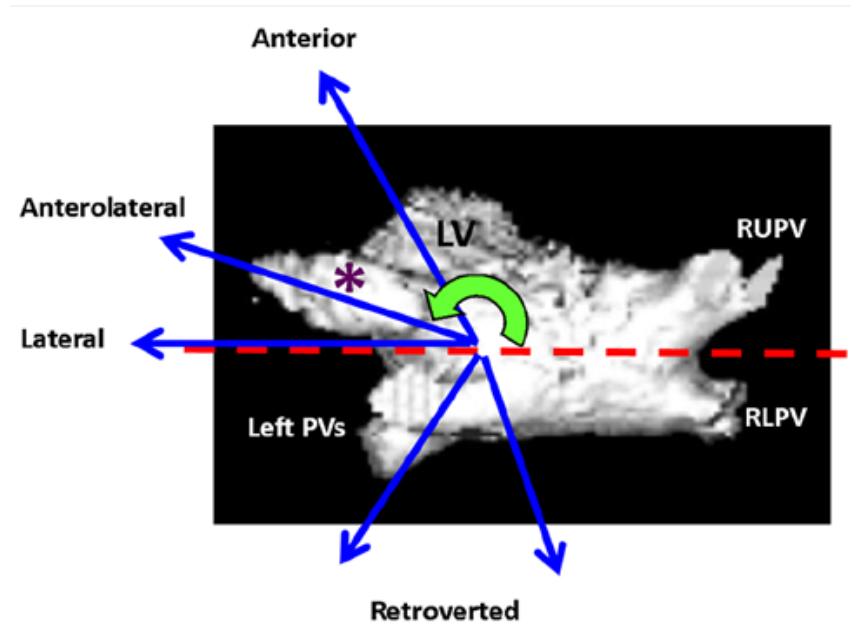

Figure 2. Three-dimensional surface-rendered image of the left atrium viewed from above (superior view) demonstrating the potential major directions of the left atrial appendage (LAA; blue arrows) relative to a coronal plane through the center of the left atrium (red dashed line). The LAA in this figure, marked by an asterisk $\left(^{*}\right)$, is in an anterolateral orientation. The green arrow indicates the angle of orientation measured from the coronal plane with zero in the right lateral position. LV, left ventricle; PVs, pulmonary veins; RUPV, right upper pulmonary vein; RLPV, right lower pulmonary vein.

tation, and lobes/lobules, rotating the image when necessary to obtain an optimal view. Further 2-D slices through relevant regions were used to determine other geometrical measures (e.g., angles) as detailed below.

\section{LAA Morphology and Orientation Ascertainment}

Shape. LAA shape was categorized into: claw-shaped, fan-shaped, cone-shaped, or s-configured (Figure 1C). Coneshaped LAAs narrowed to a tip at their distal point without any curvature, whereas claw-shaped LAAs had a single curve, and s-configured LAAs had a double curve. Fan-shaped LAAs were thin and flat, with the maximal width in one direction at least twice that in the perpendicular plane. The morphology of LAAs with complex shapes was categorized based on its closet resemblance to one of the four pre-defined morphologies. We preferred to use standard anatomical/geometric descriptions rather than pictographic descriptions (e.g., chicken wing, cactus) due to inconsistencies in these pictographic shapes.

Orientation/Angulation. The orientation of LAAs was classified according to the major direction of the tip relative to the LAA orifice in an axial plane. This orientation was defined more precisely by determining the angle between a coronal plane through the center of the left atrium and the major longitudinal axis of the LAA when viewed from above. Based on this angle, LAAs were assigned to the following categories: anteverted $\left(\leq 110^{\circ}\right)$, anterolaterally directed $\left(111\right.$ to $\left.150^{\circ}\right)$, laterally directed $\left(151\right.$ to $\left.180^{\circ}\right)$, or retroverted $\left(>180^{\circ}\right)$, with the major axis passing dorsally to a coronal section through the

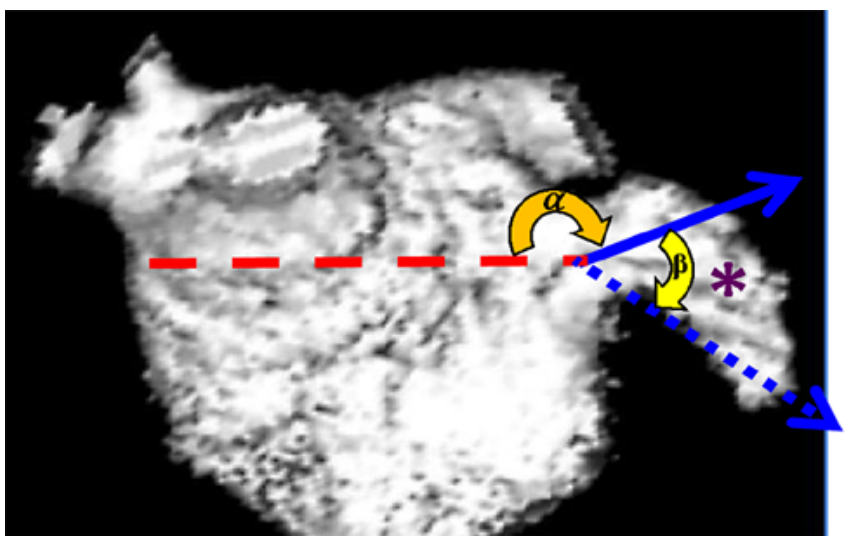

Figure 3. Left atrium viewed from the anterior (ventral) surface demonstrating the angulation of the left atrial appendage (LAA), which is marked by an asterisk $(*)$. The red dashed line indicates the horizontal (i.e., transverse) plane. The solid blue arrow indicates the direction of the proximal tubular portion of the LAA, and the orange blocked arrow indicates the angle between this and the horizontal plane (a). The blue dashed arrow indicates the line from the LAA origin to the tip, and the yellow blocked arrow indicates the change in angle between this and the solid blue arrow $(\beta)$.

LAA orifice (Figure 2). Multi-lobed LAAs (in which lobes often lie in different planes) were classified according to the orientation of the largest lobe. For twisted, multi-directional LAAs, analysis was performed in the direction of the major LAA part.

Precise measurement of LAA curvature is difficult; whereas identification of the long axis of the tubular neck is relatively easy, identification of the distal long axis is hindered by unclear tubular lines. We therefore examined the angulation of the proximal LAA neck (assessed as the angle between a transverse plane through the LAA orifice and the major longitudinal axis of the proximal LAA (angle a, Figure 3 ) in addition to measuring the change in angle between this line through the proximal LAA and a line from the orifice to the tip (angle $\beta$, Figure 3). These provide an indication of the degree of proximal angulation and curvature of the rest of the LAA. In multilobed LAAs, analysis was performed on the major lobe. The change in angle was categorized as stable $\left(0^{\circ}\right)$, mild increase $\left(1\right.$ to $\left.30^{\circ}\right)$, moderate increase $\left(31\right.$ to $\left.60^{\circ}\right)$, or severe increase $\left(>60^{\circ}\right)$, with corresponding categories for negative values (superior/retroverted angulation).

Number of Lobes and Lobules. LAAs were considered to have at least one lobe (i.e., a tubular body with a blind-ending sac). If at least one cleft split the LAA by at least $50 \%$ of its length, the regions on either side of the cleft(s) were deemed to be separate lobes (Figure 1A). Further criteria, we took into consideration, were the ones defined by Veinot et al. [9]: a visible outpouching from the main tubular body of the LAA (usually demarcated by an external crease) that was (1) occasionally but not necessarily associated with a change in direction 


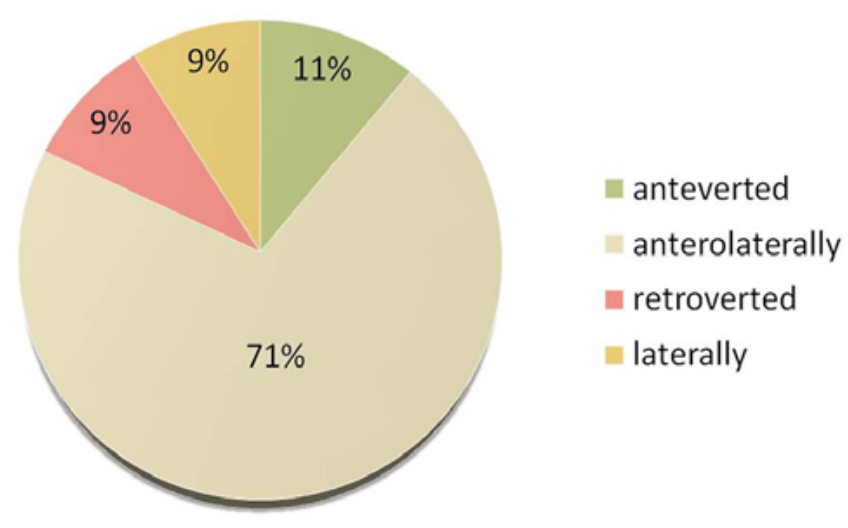

Figure 4. Distribution of different left atrial appendage (LAA) positions/orientations. Anteverted $\left(\leq 110^{\circ}\right)$, anterolaterally directed $\left(111\right.$ to $\left.150^{\circ}\right)$, laterally directed $\left(151\right.$ to $\left.180^{\circ}\right)$, or retroverted $\left(>180^{\circ}\right.$, with the major axis passing dorsally to a coronal section through the LAA orifice).

of the main tubular body of the LAA or (2) lying in a different anatomic plane to the main tubular body. Lobules were defined by a clear separate pouch from the main lobe but with a division/cleft of the lobe by less than 50\% (Figure 1B and 1D).

\section{Statistical Analysis}

Categorical data are reported as percentages, and group comparisons were performed using Fisher's exact tests. Group comparisons for continuous data were performed using Wilcoxon-Mann-Whitney $U$ tests. Normally distributed continuous variables (according to David's test) are expressed as mean \pm standard deviation (SD). In histograms, the number of class divisions was calculated by the root of the sample size. $P$-values $<0.05$ were considered to indicate statistical significance. Statistical analyses were performed using BiAS. for Windows 9.10 Software (Johann Wolfgang Goethe-University, Frankfurt, Germany).

\section{Results}

\section{Shape}

Most LAAs were claw-shaped (82\%), including all retroverted LAAs. The remaining LAAs were fanshaped $(5 \%)$, cone-shaped (8\%), or s-configured (5\%).

\section{Orientation/Angulation}

Most LAAs were anterolaterally directed when viewed from above (78\%), although a sizeable minority of LAAs were retroverted (9\%), with the remainder being anteriorly or laterally directed (Figure 4). The ranges and frequencies of orientation were similar between sexes, with a slightly higher frequency of retroverted LAAs in women (16\%) than in men (6\%), although the sample sizes were small. The angulation of the proximal LAA (angle a) ranged widely, from 77 to $160^{\circ}$ (mean, $125 \pm 16^{\circ}$ ). In $75 \%$ of individuals, angle a was between 110 and $140^{\circ}$, including the majority of retroverted LAAs ( 6 out of 7 ; Figure $5 \mathrm{~A}$ ). Almost two-thirds of LAAs (58\%) had a mild or moderate increase in curvature (angle $\beta, 1$ to $60^{\circ}$ ) between the proximal and most distal part of the LAA (Figure $5 B$ ). Most retroverted LAAs had a significant change in angle $\left(-30\right.$ to $\left.-90^{\circ}\right)$. A minority of claw-shaped LAAs had no change in angle (8\%). Likewise, all cone-shaped LAAs, by virtue of their definition, had no change in angle.

\section{Lobes and Lobules}

Of all appendages, $66 \%$ had a single lobe, $30 \%$ were bi-lobed, and $4 \%$ were tri-lobed. The mean number of lobes was $1.4 \pm 0.6$, with a range from 1 to 3 . Six of the seven retroverted LAAs consisted of one lobe, with the remaining one having two lobes. The majority of patients had at least one additional lobule (90\%), with a mean number of $2.0 \pm 1.2$ lobules (range, $0-5$ ), which tended to be located at the tip of a lobe.

\section{Discussion}

While most patients had a classical (i.e., clawshaped) LAA shape and curvature, we found significant variation in LAA orientation, shape, and curvature, with unusual shapes in $18 \%$ of patients, retroverted LAAs in approximately $10 \%$ of patients, and a wide range of angulations and multiple lobes in $90 \%$ of patients. This may have significant implications for clinical practice during LAA closure or the identification of LAA thrombus by TOE.

\section{TOE-Guided Thrombus Detection}

Despite the utility of TOE for thrombus detection in the LAA [13], a small number of thromboembolic events continue to occur even when no thrombus is detected in a pre-cardioversion [8] or LAA-occlusion setting $(1-2 \%)[5,6]$. Possible causes include absent or sub-therapeutic anticoagulation [14] and air embolism due to insufficient venting during LAA occlusion [5]. It is conceivable that thrombi may be missed during TOE examination, particularly in patients with retroverted LAAs and/or multiple lobes and large an- 

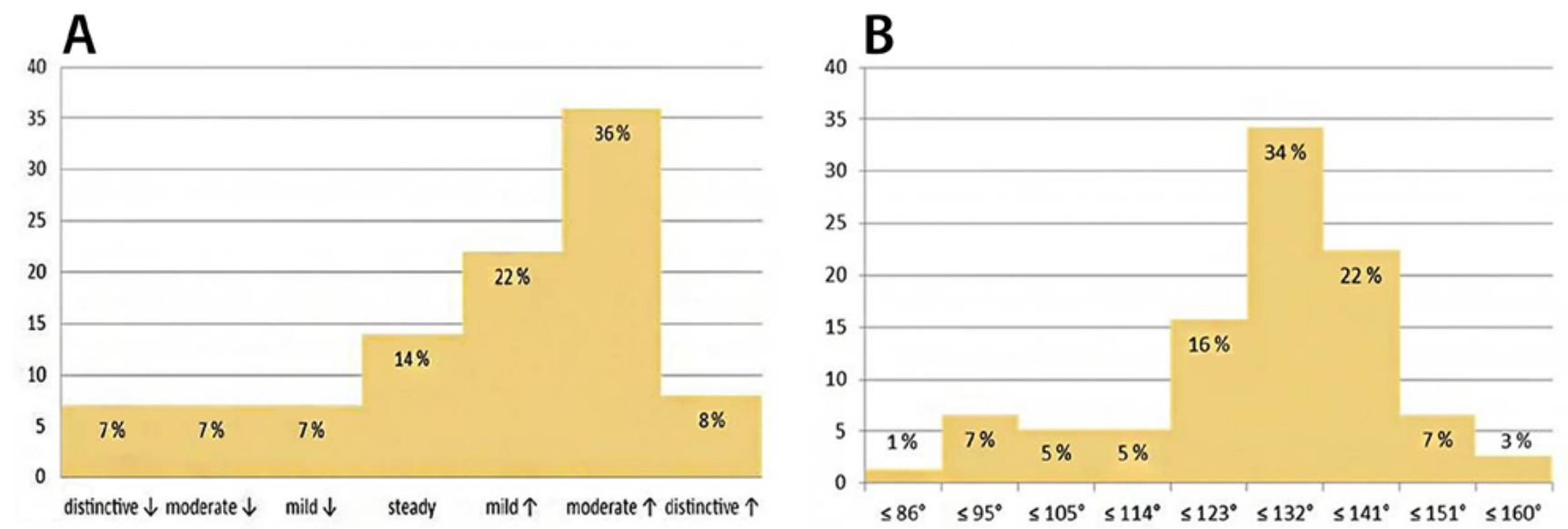

Figure 5. Panel $A$ and Panel $B$. Distribution of (Panel $A$ ) the angulation of the proximal tubular portion of the left atrial appendage $(L A A)$ to the horizontal plane (angle $a$, see Figure 3) and (Panel B) the change in LAA curvature (angle $\beta$, see Figure 3).

gulations, in whom complete imaging would require LAA interrogation in multiple views.

Although most LAAs in our study (77\%) were anterolaterally directed, retroverted LAAs were found in $9 \%$ of patients. Previous studies using computed tomography (CT) angiography, invasive angiography, and cadaveric materials have reported the presence, but not the frequency, of retroverted LAAs $[15,16]$. Some retroverted LAAs can appear relatively normal on standard views (typically mid-esophageal, with a beam angulation of $75^{\circ}$ ), with an apparent apex at the angulated bend. As a result, ensuring the identification of any retroverted lobes requires comprehensive assessment with multiple angulations of the ultrasound beam, which are not routinely employed. Our study highlights the variable LAA morphology that can complicate TOE-guided thrombus detection and emphasizes the importance of LAA imaging in all planes and angles, keeping in mind that retroverted appendages and/or multiple lobes are common. Although our findings suggest a slightly higher prevalence of retroverted LAAs among females than males, the small sample sizes limit our ability to draw firm conclusions. Further studies on this subject might be important due to the higher risk of AF-related stroke in women in the presence of other risk factors (e.g., $\mathrm{CHA}_{2} \mathrm{DS}_{2}$-VASc score) [17].

\section{Interventional LAA Closure}

The significant variation in shape, orientation, and curvature of the LAA is important for LAA closure device design and procedural technique. As the thin walls of the LAA (muscular wall $\leq 1 \mathrm{~mm}$ ) are vulnerable, device maneuvering during the procedure [18] together with anatomical variation in the LAA may partly explain the most common $(4-5 \%)[5,6]$ risk of perforation and pericardial hemorrhage. Malalignment of the delivery system with the central axis of the LAA may cause tension/stress on the LAA or may result in suboptimal device positioning, leading to more manipulations including device recapturing and redeployment.

Although no trials exist to confirm this, many operators would agree that greater manipulation during delivery system alignment and device positioning carries a higher risk of perforation and hemorrhage. Entering the LAA and subsequent occluder release require alignment along the axis of the LAA body/ proximal LAA. This angle (a) was between $110^{\circ}$ and $140^{\circ}$ in $75 \%$ of LAAs in our study, including the majority of retroverted LAAs (6 out of 7). With the current WATCHMAN LAA occlusion device, the delivery sheath must be inserted to a depth equal to at least that of the maximum ostial diameter $(21-33 \mathrm{~mm})$. In acutely angled appendages, particularly those that are retroverted, this may add to the complexity of the procedure. Similarly, the Amplatzer Cardiac Plug requires a proximal landing zone of at least $10 \mathrm{~mm}$ to deploy the distal lobe of the device. Thus, the curvature of the LAA is also important, and in $58 \%$ of cases, we observed a mild to moderate increase in angle (1 to $60^{\circ}$ ). This change in angle was greater in retrovert- 
ed LAAs, with one half showing a moderate decrease and the other showing a severe decrease in angle (-30 to $\left.-90^{\circ}\right)$. This combination of unusual direction and change in curvature may complicate deep insertion of the WATCHMAN device into the LAA. Our characterization of LAA morphology could improve delivery system design to facilitate access to the LAA and may provide guidance for catheter tip configuration, allowing smoother positioning within the LAA. These improvements have the potential to reduce the risk of perforation and pericardial hemorrhage. Optimally, multiple delivery sheaths with a variety of curvatures that approximate angulations of the appendage assessed with non-invasive imaging such as CT or MRI together with pre-procedural characterization of the LAA with respect to its angulation and curvature would minimize the need for undue sheath manipulation and device recapture maneuvers.

\section{LAA Lobes and Lobules}

Complete LAA assessment and closure is complicated by multi-lobed appendages, as lobes can exist in different planes requiring complex visualization in multiple views. Our results regarding the number of lobes are consistent with those of Heist et al. [10], who also used MRI to analyze the LAA in AF patients and found that about two-thirds of LAAs had one lobe, one-third had two lobes, and a small proportion $(<5 \%)$ had multiple lobes ( $\geq 3$ lobes). However, Veinot et al. [9] found a much higher prevalence of multiple lobes in post-mortem specimens of normal hearts, with $80 \%$ of LAAs having more than one lobe. These discrepancies among studies may arise from differences in lobe definitions and a lack of distinction between lobes and smaller variants (i.e., lobules). In addition, further extra- and intraluminal criteria (i.e., an external crease and accessibility by a probe, respectively) potentially increasing the number of lobes were taken into account by Veinot et al. but could not be assessed in our study due to different analytical methods. Finally, identification of small lobules may have been more difficult in our study due to smaller spatial resolution by non-gated MRI compared with direct visualization in post-mortem studies.

Limitations of our study include a small sample size; therefore, quoted proportions should be regard- ed as approximate. However, our study was undertaken to demonstrate the range of shapes and angulations that can occur in LAAs to avoid the assumption of uniform geometry rather than to report precise proportions of each morphology. Twenty-seven patients with inadequate image quality for LAA assessment were excluded, which in theory may have led to selection bias. However, such a bias would have required that LAA morphology affected CMR image quality, which is unlikely as this is not dependent on LAA orientation. Most importantly, we performed our analysis based on the assumption that knowledge of LAA morphology and recognition of its variants may lead to improvements in device and delivery system designs, thereby reducing the risk of procedural complications. Though plausible to operators, this assumption remains to be proven. Our study population consisted only of patients with current/paroxysmal AF, who may likely have larger left atria. Although this could alter LAA shape, significant modifications seem unlikely. Furthermore, these patients are also those in whom TOE assessment for thrombus or LAA device closure is usually performed; therefore, they are a valid group to study.

Our finding that the morphology and orientation of the LAA can vary significantly may have implications for clinical practice. Retroverted LAAs and multiple LAA lobes are common, and the shape and curvature/angulation displays wide variation. Knowledge of these morphological aspects may improve the detection of LAA thrombus on TOE and may also improve delivery sheath configuration and LAA occlusion device design to reduce procedural risk.

\section{Acknowledgements}

Saul G Myerson is funded by the Oxford National Institute for Health Research (NIHR) Biomedical Research Centre Program.

\section{Conflict of Interest}

The authors have no conflict of interest relevant to this publication.

Comment on this Article or Ask a Question 


\section{References}

1. Wolf PA, Dawber TR, Thomas HE Jr, Kannel WB. Epidemiologic assessment of chronic atrial fibrillation and risk of stroke: the Framingham study. Neurology 1978;28:973-977. DOI: 10.1212/WNL.28.10.973

2. Blackshear JL, Odell JA. Appendage obliteration to reduce stroke in cardiac surgical patients with atrial fibrillation. Ann ThoracSurg 1996;61:755-759. DOI: 10.1016/00034975(95)00887-X

3. Hart RG, Benavente O, McBride R, Pearce LA. Antithrombotic therapy to prevent stroke in patients with atrial fibrillation: A meta-analysis. Ann Intern Med 1999;131:492-501. DOI: 10.7326/00034819-131-7-199910050-00003

4. Camm AJ, Lip GY, De Caterina R, Savelieva I, Atar D, Hohnloser SH, et al. ESC Committee for Practice Guidelines (CPG). 2012 focused update of the ESC Guidelines for the management of atrial fibrillation: an update of the 2010 ESCGuidelines for the management of atrial fibrillation. Developed with the special contribution of the European Heart Rhythm Association. Eur Heart J2012;33:2719-2747. DOI: 10.1093/ eurheartj/ehs253

5. Reddy VY, Sievert H, Halperin J, Doshi SK, Buchbinder M, Neuzil P, et al. PROTECT AF Steering Committee and Investigators. Percutaneous left atrial appendage closure vs warfarin for atrial fibrillation: a randomized clinical trial. JAMA 2014;312:1988-1998. DOI: 10.1001/jama.2014.15192

6. Park JW, Bethencourt A, Sievert $H$, Santoro G, Meier B, Walsh K, Lopez-Minquez JR, et al. Left atrial appendage closure with Amplatzer cardiac plug in atrial fibrillation: initial European experience. Catheter Cardiovasc Interv 2011;77:700-706. DOI: 10.1002/ ccd. 22764

7. Viles-Gonzalez JF, Kar S, Douglas P, Duk- kipati S, Feldman T, Horton R, et al. The clinical impact of incomplete left atrial appendage closure with the Watchman Device in patients with atrial fibrillation: a PROTECT AF (Percutaneous Closureof the Left Atrial Appendage Versus Warfarin Therapy for Prevention of Stroke in Patients With Atrial Fibrillation) substudy. J Am Coll Cardiol 2012;59:923-929. DOI: 10.1016/j.jacc.2011.11.028

8. Black IW, Fatkin D, Sagar KB, Khandheria BK, Leung DY, Galloway JM, et al. Exclusion of atrial thrombus by transesophageal echocardiography does not preclude embolism after cardioversion of atrial fibrillation. A multicenter study. Circulation 1994;89:2509-2513. DOI: 10.1161/01. CIR.89.6.2509

9. Veinot JP, Harrity PJ, Gentile F, Khandheria $\mathrm{BK}$, Bailey KR, Eickholt JT, et al. Anatomy of the normal left atrial appendage: a quantitative study of age-related changes in 500 autopsy hearts: implications for echocardiographic examination. Circulation 1997;96:3112-3115. DOI: 10.1161/01. CIR.96.9.3112

10. Heist EK, Refaat M, Danik SB, Holmvang G, Ruskin JN, Mansour M. Analysis of the left atrial appendage by magnetic resonance angiography in patients with atrial fibrillation. Heart Rhythm 2006;3:1313-1318. DOI: 10.1016/j.hrthm.2006.07.022

11. Di Biase L, Santangeli P, Anselmino M, Mohanty $P$, Salvetti I, Gili S, et al. Does the left atrial appendage morphology correlate with the risk of stroke in patients with atrial fibrillation? Results from a multicenter study. J Am Coll Cardiol 2012;60:531-538. DOI: 10.1016/j.jacc.2012.04.032

12. Lacomis JM, Goitein O, Deible C, Moran PL, Mamone G, Madan S, et al. Dynamic multidimensional imaging of the human left atrial appendage. Europace 2007;9:11341140. DOI: 10.1093/europace/eum227

13. Wheeler R, Masani ND. The role of echocardiography in the management of atrial fibrillation. Eur J Echocardiogr 2011;12:133138. DOI: 10.1093/ejechocard/jer124

14. Khan IA. Transient atrial mechanical dysfunction (stunning) after cardioversion of atrial fibrillation and flutter. Am Heart J 2002;144:11-22. DOI: 10.1067/ mhj.2002.123113

15. Sievert H, Qureshi SA, Wilson N, Hijazi ZM. Percutaneous Interventions for Congenital Heart Disease. Informa Healthcare 2007; 1st edition:582. DOI: 10.3109/9780203018262

16. Ho SY, McCarthy KP. Anatomy of the left atrium for interventional electrophysiologists. Pacing Clin Electrophysiol 2010;33:620-627. DOI: 10.1111/j.15408159.2009.02659.x

17. Lip GY, Nieuwlaat R, Pisters R, Lane DA, Crijns HJ. Refiningclinical risk stratification for predicting stroke and thromboembolismin atrial fibrillation using a novel risk factor-based approach: The euro heart survey on atrial fibrillation. Chest 2010;137:263272. DOI: $10.1378 /$ chest.09-1584

18. Su P, McCarthy KP, Ho SY. Occluding the left atrial appendage: anatomical considerations. Heart 2008;94:1166-1170. DOI: 10.1136/hrt.2006.111989

Cite this article as: Joy $\mathrm{S}$, Sievert $\mathrm{H}$, Bertog S, Betts T, Wilson N, Myerson S. Left Atrial Appendage Morphology in Patients with Non-Valvular Atrial Fibrillation. Structural Heart Disease. 2017;3(1):8-14. DOI: http://dx.doi. org/10.12945/j.jshd.2017.16.003 